\title{
Riflessioni e commenti sull'“'Essai sur la formation du dogme catholique" di Cristina di Belgiojoso (e le relazioni della Sacra Indicis Congregatio)
}

\section{Annarosa Poli}

\section{(2) OpenEdition}

\section{Journals}

Edizione digitale

URL: http://journals.openedition.org/studifrancesi/3607

DOI: 10.4000/studifrancesi.3607

ISSN: 2421-5856

\section{Editore}

Rosenberg \& Sellier

\section{Edizione cartacea}

Data di pubblicazione: 1 décembre 2012

Paginazione: 477-503

ISSN: 0039-2944

\section{Notizia bibliografica digitale}

Annarosa Poli, «Riflessioni e commenti sull"Essai sur la formation du dogme catholique" di Cristina di Belgiojoso (e le relazioni della Sacra Indicis Congregatio)», Studi Francesi [Online], 168 (LVI | III) | 2012, online dal 30 novembre 2015, consultato il 05 mars 2021. URL: http://journals.openedition.org/ studifrancesi/3607 ; DOI: https://doi.org/10.4000/studifrancesi.3607

\section{(c)}

Studi Francesi è distribuita con Licenza Creative Commons Attribuzione - Non commerciale - Non opere derivate 4.0 Internazionale. 


\section{Riflessioni e commenti \\ sull" Essai sur la formation du dogme catholique" di Cristina di Belgiojoso (e le relazioni della Sacra Indicis Congregatio)}

\section{Una principessa scrittrice, liberale, patriota}

Cristina Trivulzio, dotata di un fascino notevole, nobile, intelligente, insofferente dell'oppressione straniera, non cesserà per tutta la vita di affermare le sue convinzioni con l'intento di sovvertire l'ordine del suo tempo.

Nata all'inizio del secolo, nel 1808, rimasta orfana del padre a 4 anni, riceve un'educazione tradizionale dell'aristocrazia lombarda che richiedeva una buona conoscenza delle lingue, in particolare del francese, qualche nozione del disegno, musica e canto, di geografia, algebra e geometria.

Ma la sua vera educazione la farà da sola, leggendo testi in italiano, inglese e francese, e frequentando personaggi tra i più notevoli della cultura del suo tempo.

La giovinetta era cresciuta raccolta in una vita tutta interiore, l'aria che respirava in casa sua, luogo di riunioni dei liberali più noti, attorno al suo patrigno, il marchese Alessandro Visconti d'Aragona, farà nascere in lei i primi germi di patriottismo.

Dopo l'arresto del patrigno da parte della polizia austriaca, le angosce della madre e dei fratelli durante la lunga detenzione in attesa della sentenza, suscitano in lei l'odio contro l'Austria e la spingono a dedicarsi tutta alla realizzazione dell'indipendenza dell'Italia.

Apparentemente fredda, anche perché spesso sofferente nel fisico, era dotata di una volontà ferrea e di una fierezza orgogliosa.

La sua insegnante di disegno, Ernesta Bisi, la introduce nel mondo dei cospiratori di Milano. Decide, a soli 16 anni, di sposare il principe Emilio di Belgiojoso, anche lui fervido patriota, ma uomo che le viene sconsigliato da tutti i suoi amici.

Nel 1828 ottiene una separazione consensuale dal marito, che la trascura e la tradisce. Amareggiata e infelice comincia a viaggiare e a frequentare a Genova case patrizie di patrioti. L'Austria segue i suoi movimenti.

Da Lugano, dove si è rifugiata nel 1831, è costretta a trasferirsi in Francia, e vi trova aiuti per sostenere la sua lotta politica. Entra a far parte delle dame di corte della regina e fonda un salotto che sarà molto frequentato perché simboleggia l'avvenire. Nel 1836 però sarà costretta a chiuderlo.

In seguito alla concessione dell'amnistia ai fuoriusciti, avverte la necessità di creare un legame tra la sua vita intima e quella che stava conducendo. Ha sempre sentito il desiderio di dedicarsi agli altri e possiede un senso vivo della fratellanza. Incontra un giovane abate, l'Abbé Cœur, e trova in lui un'interiore corrispondenza alle sue esigenze spirituali, da lungo tempo in crisi nel fondo del suo animo.

Il marito è tornato vicino a lei e così presto anche il desiderio di maternità potrà avverarsi. Nel 1838 nascerà Maria Gerolama di Belgiojoso.

Nel 1840, a Parigi, Cristina comincia a scrivere l'Essai; nello stesso anno, decide di ritornare in Italia e ritirarsi nella sua proprietà di Locate Triulzi, dove apre un asilo ed una scuola, ispirandosi a Fourier.

Il suo comportamento troppo socialistezzante viene criticato dall'aristocrazia milanese che condanna queste idee per paura che costituiscano una minaccia per le loro proprietà private. 
Lo stesso Alessandro Manzoni le impedirà di assistere sua madre, Giulia Beccaria, ormai moribonda, che era una sua intima amica.

A Locate fa costruire nuove abitazioni e cerca di diffondere il benessere tra $\mathrm{i}$ tanti poveri del luogo. Nel frattempo continua la stesura del suo Essai.

Durante un breve soggiorno a Parigi incontra Alfred de Musset che è sempre stato innamorato di lei e mai corrisposto, anche se la principessa si dimostra un'amica fraterna prestandogli aiuto in un momento difficile. Il poeta non le perdonerà mai il suo rifiuto amoroso.

In quei giorni il marito Emilio la lascerà per seguire la giovane duchessa di Plaisance.

Nel 1844 torna a Locate, pubblica la traduzione francese de La scienza nuova di G.B. Vico e contribuisce, con una cospicua forma di denaro, a creare «La Gazzetta Italiana», stampata all'estero e diffusa in Italia per richiamare l'attenzione degli italiani sul problema sociale.

La polizia austriaca ne impedisce la distribuzione e lei crea la rivista «L'Ausonio», per far conoscere la condizione morale-politica-finanziaria del suo Paese. Per maggior sicurezza ne sposta la sede a Napoli, dove si reca, festeggiata durante il viaggio da Firenze a Roma.

Nel marzo 1848 Milano è libera dagli Austriaci. La coraggiosa nobildonna vi ritorna, accompagnata dai patrioti meridionali.

Nell'agosto del '48 gli Austriaci riprendono il possesso della città. Cristina, impavida, continua a lottare per la liberazione dell'Italia dallo straniero.

Nell'aprile 1849, dopo la sconfitta piemontese, decide di recarsi nella Repubblica romana in cui vede la possibilità di costruire un primo stato italiano indipendente e si riunisce a Mazzini.

A Roma le viene affidata la direzione delle ambulanze civili e militari e organizza anche diversi ospedali che saranno preziosi per i feriti di guerra.

Nel 1849, dopo la vittoria dei francesi, deve lasciare l'Italia e si rifugia prima a Malta, poi ad Atene ma, non essendo accolta favorevolmente, si sposta a Scutari. Là scriverà le sue impressioni in La vita intima e la vita nomade in Oriente, ed un romanzo, Emina, pubblicati successivamente nel 1855 e nel 1856.

Nel 1853 fa un viaggio a Gerusalemme. Ora che ha finalmente trovato un po' di serenità, viene aggredita da un servo con un colpo alla nuca che la obbligherà a restare con la testa piegata per il resto della sua vita.

Nel 1861 è rimasta sola. La figlia si è sposata ed il marito, fuggito, era morto nel 1858.

Nel 1866 scrive in «Nuova Antologia» un saggio Della presente condizione delle donne e del loro avvenire.

Nel giugno del 1871 di ammala di una forma epatica e muore a Milano il 5 luglio.

\section{Il pensiero politico e filosofico di Cristina di Belgiojoso nell' "Essai sur la formation du dogme catholique"}

Cristina, come detto, inizia a scrivere in francese la sua opera a Parigi nel 1840 (la completerà a Locate): quattro volumi che saranno stampati tra il 1842 ed il 1843, a Parigi, senza il nome dell'autore, e subito messi al bando dalla Chiesa di Roma con un Decretum del 17 agosto $1843^{1}$. In essa chiedeva il libero esame, proponeva una Chiesa meno bloccata nella reazione e si soffermava sulla dottrina di Origene, allora non proibito ad alcune personalità del clero, ma con l'avvertimento "cave Originem".

(1) Qui allegato in due documenti della Sacra Indicis Congregatio. 
La Belgiojoso non propone soluzioni, ma piuttosto dei problemi: ogni personaggio di cui indaga il pensiero viene proposto con l'interrogativo del dubbio dialettico.

La scrittrice sviluppa il pensiero cristiano partendo dal II secolo d.C., soffermandosi sulla lotta tra arianesimo e cristianesimo e sull'avvento dell'imperatore Teodosio e termina con la storia dei regni franchi e lombardi, l'Impero di Bisanzio e la politica del papato. Quando il cristianesimo cominciò a propagarsi, grazie agli apostoli e ai loro seguaci, fu costretto a dover combattere contro il paganesimo e inoltre contro la filosofia alessandrina e le teogonie orientali. Queste ultime, agendo su un fondo culturale spiritualmente impegnato, pensarono di adattare le lezioni evangeliche alle esigenze magiche delle loro dottrine. Così al Cristianesimo toccò di essere alterato da influssi paganeggianti, soprattutto neoplatonici, e sconvolto dalle fantasiose teogonie orientali.

Nel primo caso ci troviamo di fronte a personalità di eccezionale cultura come Filone di Alessandria; nel secondo a personalità ricche di conoscenza delle supreme volontà. Questa realtà culturale così variegata assumeva colori diversi, in relazione alla scuola seguita da ciascun filosofo.

Si possono infatti individuare tre centri culturali: Alessandria, Cartagine e Roma.

Su questo piano, dentro al quale si muoveva vivacemente il pensiero filosofico, è possibile individuare il meccanismo che dà vita al formarsi dei dogmi: una corrente di pensiero cristiano che si sviluppa su una dottrina, basata su principi filosofici laici, nella convinzione di arricchire il testo evangelico o di offrire nuovi mezzi per l'interpretazione o l'esegesi dei testi biblici; oppure una corrente di pensiero, sia pur cristiano, che riteneva di poter fondare le sue verità su misteri orfici, su credenze pitagoriche o su sistemi surreali.

Sia in un caso che nell'altro, coloro che si attenevano scrupolosamente al testo evangelico accusavano di eresia le nuove dottrine, combattendole fino al momento in cui non fosse stato deciso di riunire i vescovi in un Concilio o in un Sinodo. La discussione della nuova dottrina conduceva sia alla sua accettazione, sia al suo rifiuto: in tutti e due i casi ne derivava una decisione identificabile con un canone che aveva la forza di un dogma. Il procedimento è sempre lo stesso: eresia $\rightarrow$ reazione ortodossa Concilio o Sinodo $\rightarrow$ dogma.

C'è da stupirsi di come Cristina affronti l'ampia e complessa problematica così sinteticamente semplificata.

La scrittrice parte dall'esame della dottrina di ogni singola figura che si accinge a studiare, esaminando personalità che occupano una posizione notevole nella storia del pensiero cristiano, ampliando progressivamente il discorso fino a presentarne altre che gravitano attorno alle loro opere, giungendo a disegnare, dove è possibile, il quadro dell'umanità contemporanea ai fatti narrati.

La prima figura di cui si occupa è Ireneo, di cui traccia una fisionomia umana che rimbalza poi dall'esame delle sue opere e delle sue azioni attraverso i secoli fino ai suoi giorni: «L'un des pères de l'Église qui ont établi d'une manière précise et aussi claire que possible les dogmes fondamentaux du Christianisme» ${ }^{2}$, scrive Cristina.

Nell'opera di Ireneo, Contro le eresie, si può comprendere chiaramente come il dogma si affermi nella lotta dell'ortodossia contro la credenza cosiddetta "eretica"; non solo, ma Cristina attingendo e confutando fonti, cioè le storie della Chiesa di Eusebio di Cesarea e di Sonzomene e l'opera stessa di Ireneo, offre un quadro 
drammatico dell'affermarsi dell'ortodossia contro l'eresia di Montano. L'autrice segue il formarsi e lo svilupparsi del pensiero eretico sui libri di Ireneo, di Epifanio, di Teodoreto. Ha presente il Panarion di Epifanio nel tratteggiare le dottrine eretiche.

Quando, nel secondo capitolo, tratta di Clemente di Alessandria, Cristina ricorre agli strumenti del reportage: ricreare sulle orme di Thierry, attraverso lo stesso discorso del protagonista, l'ambiente dove si muove la realtà sociale in cui è immerso. In un lungo passo del Pedagogo «le plus savant de tous les auteurs ecclésiastiques»3, descrive i costumi poco severi dei primi cristiani, donne e uomini, allontanandosi dalla sua funzione di informazione per poi trasformarlo in una pagina di contenuto storico.

Nell'esaminare gli Stomata, Cristina sintetizza in una frase la problematica culturale e spirituale di Clemente: «les philosophes préparent à la foi sur laquelle est fondée la science» ${ }^{4}$.

Ma in realtà, il vero protagonista della prima parte del volume I è Origene, a cui Cristina dedica le pagine più appassionate. Origene fu una delle figure più discusse dalla Patrologia, tanto che l'atteggiamento di Cristina dovette suscitare non poche polemiche ed essere considerato sul limite tra ortodossia ed eresia 5 .

L'esegesi allegorica dei libri testamentari, troppo avveniristica per quei tempi, e la profonda cultura, posero ben presto Origene in conflitto con i vescovi, soprattutto con Demetrio, vescovo di Alessandria.

La domanda che Cristina si pone, circa l'opportunità di convocare un Concilio che giudichi finalmente l'opera di Origene è la seguente: «Disons encore que si les adversaires d'Origène pouvaient compter plus de trois chefs, que si ces chefs étaient recommandables par leur prudence, leur impartialité et leur équité, nous serions encore en droit d'écouter Origène et de demander, pour lui fermer nos oreilles et notre cœur, l'ordre d'un concile œcuménique»'.

E, più tardi, risponderà a Cristina il Concilio Vaticano II.

Passando all'esame della personale interpretazione critica dei testi sacri da parte di Origene, occorre per Cristina chiarire i principi interpretativi ai quali si ispirava: «Tous les passages, dit-il, de l'Ancien au Nouveau-Testament qui, pris littéralement, nous présentent un sens absurde ou indignes de Dieu doivent être entendus spirituellement» ${ }^{\top}$.

Dopo aver esaminato l'opera di Tertulliano come rappresentante della scuola occidentale, Cristina prende in esame le condizioni della chiesa nel II e nel III secolo. La sua ricerca si sviluppa tanto da cercare di riuscire a cogliere lo slancio dell'umanità verso il suo sviluppo civile. La scrittrice esce dall'esame del pensiero individuale per ampliarsi verso il progresso dell'umanità: «En examinant l'histoire du christianisme, on voit les événements se succéder, le théâtre s'agrandir et les acteurs se multiplier rapidement. Elle ne demeure que peu de temps enfermée dans la géographie de quelques hommes; elle comprend bientôt la marche et le développement de l'esprit humain dans une de ces plus importants fonctions, l'établissement d'une doctrine religieuse» ${ }^{8}$.

Dopo aver presentato la vita solitaria di singoli eremiti, Cristina si sofferma sul pensiero di Ario e conclude: «En reconstituant les troubles dont Arius fut la cause, je n'ai pas cru devoir suivre l'exemple des historiens de l'Église qui tous s'accordent à

(3) Ibid., p. 43.

(4) Ibid., p. 64.

(5) Cfr. F. Pizzolato-M. Rizzi (a cura di), Origene, Maestro di vita spirituale, Milano, Vita e pensiero, 2001.
(6) C. Di Belgiojoso, Essai sur La Formation du dogme catholique., cit., p. 116.

(7) Ibid., p. 104.

(8) Ibid., p. 148. 
présenter ces hérésiarques sous les traits les plus odieux. Je n'ai vu dans Arius qu'un esprit novateur, mais soumis, égaré, mais repentant. Je ne saurais d'ailleurs me défendre d'un sentiment pour ces hommes qui vivent pour l'intelligence, qui emploient toute leur activité et leur force au service d'une idée qu'ils croient fondée, et qui placent leurs expériences et leur bonheur dans son triomphe et son établissement dans le monde»?.

E aggiunge: «Incapables que nous sommes de connaître la vérité absolue, nos efforts plus ou moins impuissants pour l'atteindre, nous sont comptés» ${ }^{10}$.

Così si può sintetizzare il pensiero della scrittrice: porlo.

1. Se il pensiero vuole assicurarsi della verità è opportuno formularlo e non im-

2. "L'esprit de vérité" dovrà manifestarsi come ipotesi di verità invece di imporsi dogmaticamente.

3. Il cammino verso Dio è un'elevazione dello spirito anche se non riuscirà a raggiungere la sua meta.

L'originalità di Cristina sta nella proposta di una dottrina non come verità, ma come ipotesi di verità. Da questo punto di vista anche l'eresia di Ario acquista maggior rispetto di quanto le invettive degli spiriti ecclesiastici e del suo avversario Atanasio abbiano dimostrato verso di lui.

È qui che le opinioni di Cristina divergono da quelle della chiesa proprio perché la chiesa ha voluto imporre la sua ipotesi di verità come verità svelata senza permettere una discussione in proposito. E prospetta altrimenti la possibilità che la dottrina di Cristo sia diventata la dottrina cristiana per un concorso fortuito di circostanze che in altra situazione avrebbe potuto passare inosservata come una dottrina di una setta ebraica.

L'eresia sta di fronte all'ortodossia come l'illuminazione della ragione rispetto alla nebulosità magica dei dogmi.

Trattando della reazione pagana al cristianesimo, Cristina mette in evidenza il carattere pragmatico di S. Ambrogio: «Ainsi entraîné pour son caractère à établir la puissance spirituelle au-dessus de la puissance temporelle et par son esprit à embrasser la charité, représentant actif et défenseur d'une grande révolution et d'un sublime perfectionnement, il remplit avec constance et sans hésiter sa double mission» ${ }^{11}$.

A proposito del personaggio di Gerolamo, Cristina non pare approvare il pensiero della Chiesa sulla sua santità ed esprime un giudizio severo sul Cristianesimo: «À mesure qu'en s'éloignant de son berceau, le christianisme acquiert la puissance et les richesses du monde, on le voit perdre en partie son caractère divin et tomber dans l'humanité»' ${ }^{12}$.

Gerolamo ha il merito di aver arricchito la chiesa latina di ottime traduzioni che riportavano un'interpretazione della Bibbia più vera rispetto alle precedenti, ma gli rimprovera di non aver compreso l'ideologia della scuola alessandrina e di aver accusato di eresia l'opera di Origene.

Seguendo i principi sostenuti dalla chiesa, egli non ha compreso il metodo interpretativo allegorico di Origene, più libero, meno coercitivo e così ammette: «Dans l'histoire des Juifs, il faut croire en même temps à la vérité des faits et à leur sens prophétique. Dans le dogme chrétien il faut admettre l'éternité des peines et la résurrection des corps, en même temps qu'il faut croire à la sagesse, à la toute-puissance et à la souveraineté et à la bonté de Dieu, parce-que nous l'ordonnons ainsi»» ${ }^{13}$.

(9) Ibid., pp. 380-381.

(10) Ibid., vol. I, p. 315.

(11) Ibid., vol. II, p. 85.
(12) Ibid., vol. II, p. 87.

(13) Ibid., vol. II, p. 136. 
C'è da chiedersi, sostiene Cristina, se Gerolamo capì che si trovava di fronte ad una scelta socio-politica culturale, oppure perché non comprendeva l'esegesi di Origene?

Qualunque sia la risposta, secondo Cristina, la Chiesa di Roma accettando la interpretazione storico-letterale e rafforzandola con il dogma pose la fede al di fuori d'ogni sviluppo e la rese immobile.

Ne consegue che la Vulgata è solo l'interpretazione di un testo che per volere di Gerolamo, rinforzato dal dogma della Chiesa, ha dovuto essere accettato da tutto il mondo cristiano anche in quei luoghi che non erano d'accordo con questa interpretazione.

Così Cristina conclude: «Il contribua puissamment par ses traductions, ses ouvrages, ses amis et ses erreurs même, à faire naître et à terminer la grande querelle contre l'Orient et l'Occident. Il semblait avoir quitté la terre latine et s'être transporté en Asie pour imposer à celle-ci les habitudes positives et littérales de l'esprit occidental. Il fut un de ceux qui enchaînèrent le génie de l'Orient ou qui l'obligèrent au moins à se jeter en dehors du christianisme» ${ }^{14}$.

Affrontando l'esame del pensiero di Agostino non si limita ad interpretarne le idee, ma sviluppa il discorso in una sintesi storica delle discussioni e delle controversie relative ai concetti di libero arbitrio, di predestinazione, di grazia, sia come dogma sia come basi di ricerche etico-filosofiche.

Questi tre temi diventano la parte centrale dell'opera del santo e offrono alla scrittrice il modo di scrivere le sue più belle pagine di storia ecclesiastica. Ampliando la sua indagine ad una sintesi di storia e filosofia dopo aver preso in esame l'opera di Lucido, Gatescalo, di Bredwardine, attraverso i secoli per giungere a Wicleff, Jacobez, Huss, Lutero, Calvino, fino al Concilio di Trento, così conclude: «Les Luthériens, et plus encore les Zuingliens, avaient donné la mesure de l'abîme où l'on pouvait tomber en suivant de trop près et avec trop de confiance Saint Paul et Saint Augustin» ${ }^{15}$.

A questo punto Cristina introduce il discorso sui Longobardi, il cui regno, anticipando i tempi, acquista per lei un valore positivo. Parte da un'osservazione interessante: lo stabilirsi del cristianesimo ha dato frutti imperituri ${ }^{16}$ come era generalmente riconosciuto dagli storici, ma, secondo lei, sarebbe stato più saggio dire che la chiesa aveva conservato sé stessa in mezzo al disfacimento generale da parte dei barbari imponendosi al di sopra di tutto con la sua organizzazione amministrativa e con la sua ideologia legata al dogma. Il magistero ecclesiastico opponeva la forza del pensiero che era legata alla tradizione romana, e la dottrina cristiana riuscì a dominare l'arianesimo dei Longobardi conservando un ascendente sociale.

Partendo da queste osservazioni la scrittrice tratta insieme con agile disinvoltura di quattro argomenti: il regno dei Longobardi, il regno dei Franchi, l'Impero romano e la politica del Papato.

Sostiene, allontanandosi dalla tradizione, che molti istituti giuridici longobardi si erano trasformati a contatto con il diritto romano, opinione ripresa dagli storici contemporanei, e non hanno distrutto ogni libertà o ogni diritto dei conquistatori sui Romani.

Ė evidente che si era ispirata alle dottrine del Romagnosi ${ }^{17}$ e del Savigny ${ }^{18}$.

Alessandro Manzoni, fin dal 1822, era stato di diversa opinione sostenendo che:

(14) Ibid., vol. II, pp. 142-143.

(15) Ibid., vol. II, pp. 319-320.

(16) Ibid., vol. III, p. 307.

(17) G. D. Romagnosi, Dell'indole e dei fattori dellincivilimento, Firenze, Piatti Editore, 1844, p.te II, cap. III, p. 3.

(18) F. SAvignY, Storia del diritto romano nel medioevo, Milano, 1859, p. 225. 
«Tutti i barbari che, riuniti in un corpo di nazione, si gettarono su qualche parte dell'Impero romano avevano delle leggi proprie, non scritte, ma tradizionali... Ora queste leggi che erano il loro lavoro, la loro proprietà, perché le avrebbero essi comunicate ai vinti? Ecco perché i Longobardi, come gli altri barbari, non forzarono i Romani a ricevere le leggi loro... Posti in salvo i privilegi della conquista, le relazioni tra conquistati e conquistatori diventarono indifferenti ai padroni» ${ }^{19}$.

Su questo capitolo la Belgiojoso non si è lasciata fuorviare dall'autorità del Manzoni, convinta della sopravvivenza di una civiltà romana sotto il dominio dei Longobardi. Ha messo inoltre in rilievo il rapporto tra diritto morale da cui desume l'ethos del popolo, considera l'ordinamento giuridico romano uno strumento di oppressione della libertà popolare e ha visto nel clero la funzione di collante tra il diritto romano e quello che sarà il diritto statutario. E qui Cristina espone chiaramente i limiti della sua fede: adesione formale all'autorità della Chiesa, ma libertà di mantenere l'autonomia critica. Libertà di coscienza che è anche libertà di aderire al magistero della Chiesa. Da un lato vorrebbe essere cristiana, dall'altro afferma la sua indipendenza di giudizio.

La sua fede ha un limite, crede nelle parole di Gesù che la Chiesa ha deformato attraverso il linguaggio dei papi. Da questo punto di vista si sente eretica e ipocrita e quando parla del verbo credere, esprime una speranza e non una certezza.

Dalle pagine dell'Essai emana una critica serrata alle forze che vorrebbero impedire alla ragione di esercitare la sua vera funzione, la libertà di coscienza a cui lo stesso Paolo VI ha fatto riferimento nella sua prima enciclica ${ }^{20}$.

Ma un'opera di questo genere non poteva essere approvata dalla Congregazione dell'Indice nonostante le affermazioni iniziali della Belgiojoso che dichiara all'inizio del volume: «Pénétré du plus profond respect non seulement pour le monde chrétien, mais pour l'institution divine et pour la conduite inspirée de l'église catholique, j'aurais mal rendu ma pensée si chaque mot de ce livre n'exprimait la soumission la plus entière à ses décrets. J'ai vu avant même de connaître» ${ }^{21}$. Ne era veramente convinta?

Quanto segue dell'opera sarà poi biasimato dal censore: «Mais, en lisant l'histoire de l'établissement de notre religion, en apercevant les traces du rare génie des premiers Pères, et celle de la prudence des églises, en voyant ceux-là choisir parmi les croyances vieillies les parcelles de vérité que Dieu a accordées de tout temps à l'humanité, les épurer, les rassembler et leur donner le complément nécessaire; et celles-ci, appelées à juger du mérite de ces travaux, agir avec une sagesse constante, et ne se prononcer jamais vainement, ni avec précipitation, j'ai quitté la foi aveugle pour recevoir, de la connaissance, une foi non moins entière».

Padre De-Lacroix trova inesatta l'asserzione di Cristina relativa ai giudizi delle chiese invitate a giudicare le vecchie credenze: lo fecero con saggezza e non con una certa precipitazione? Lo fece la Chiesa universale, non certo le chiese particolari.

E non sembra credere neppure all'asserzione: «Quelle que soit ma répugnance à parler encore de moi, je dois dire quoique cela me semble superflu, que nos propres opinions, renfermées dans les limites que l'Eglises a posées, ne se maintiendraient pas un seul instant devant les arrêts que l'Eglise pourrait rendre encore. Si quelque chose dans ce livre, a pu paraître signifier le contraire, je le rejette dès à présent» ${ }^{22}$.

E il censore commenta: «Lasciando adunque a parte l'intenzione dell'Autore e conservando soltanto l'opera in sé stessa, io la trovo assai difettosa, e scritta come da

(19) A. Manzoni, Discorsi sulla storia longobarda in Italia, Mondadori, 1863, cap. III, p. 224.

(20) Paolo VI, Ecclesiam suam, 1964, $\mathbb{} 11$.
(21) C. Di Belgiojoso, Essai sur La Formation du dogme catholique., cit., vol. I, p. II.

(22) Ibid., p. IV. 
persona, cui mancano le più necessarie cognizioni per trattare accuratamente materie teologiche, anzi sembrami che si deve attribuire alla mancanza stessa di tali cognizioni quella specie di presunzione colla quale l'autore parla così francamente delle questioni le più difficili, come si vedrà da alcuni passi dell'opera, che riferirò in appoggio della mia censura». che passo.

Aveva infatti precedentemente dichiarato di non aver letto l'opera, ma solo qual-

In una breve biografia di Cristina, che apre la sua relazione ${ }^{23}$, l'aveva definita «persona di molto spirito ed istruzione», aggiungendo: «Non può dirsi che sia irreligiosa, sebbene in religione come pure in politica, abbia sovente dato prova di capo alquanto stravolto».

È tuttavia convinto che questo testo non sia poi tanto pericoloso, perché passato sotto silenzio: «Quanto all'opera stessa, sebbene a parer mio meriti di essere riprovata, ciò nondimeno poiché non sembra fare alcun rumore, non le darei quella specie di importanza che risulta da una solenne e pubblica condanna, bensì per togliere dalla mente dell'autore la voglia di comporre altre opere, l'avviserei che il motivo per cui non viene vietata ai fedeli la lettura di questo suo primo lavoro è appunto quello, perché di fatto essi non lo leggono».

In realtà Padre De-Lacroix si sbagliava perché il libro ebbe subito un'eco vastissima, come vedremo in seguito. 
Essai sur la formation du dogme catholique

in una relazione del censore padre Agostino De-Lacroix, della Congregazione dell'Indice Ecclesiastico (28 gennaio 1843)

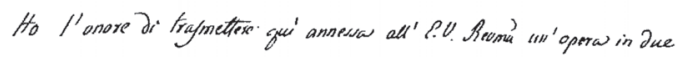

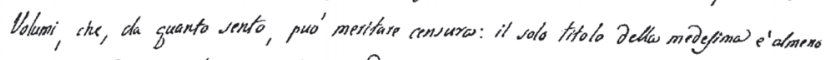

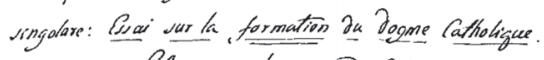

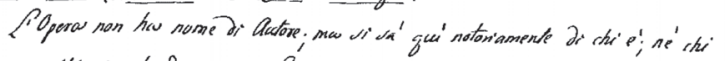

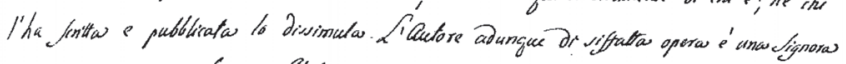

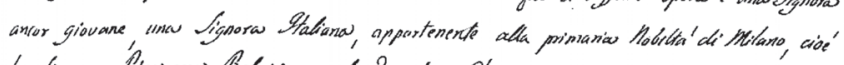

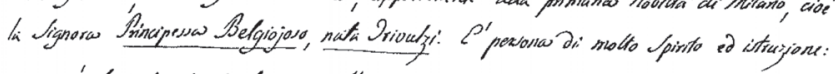

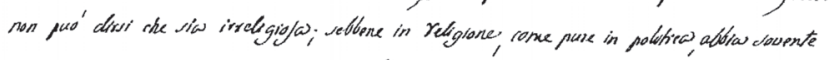

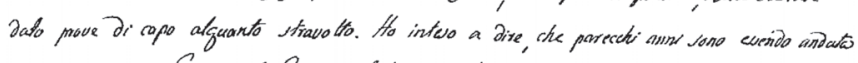

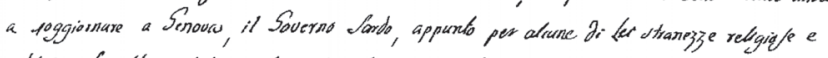

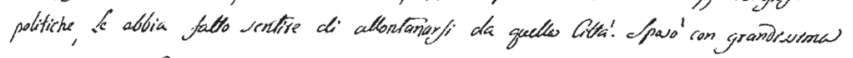

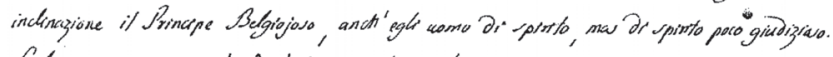

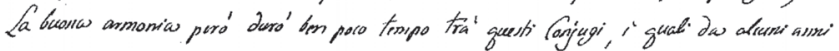

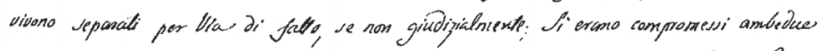

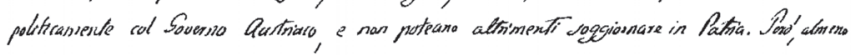

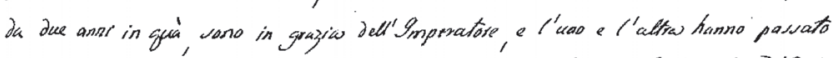

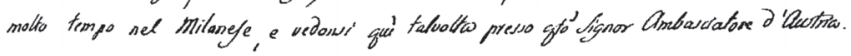
a Suw Iminerzas Riromá, It Signox Cardinal Sirustrouiond,

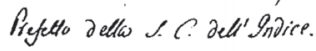

Roma.

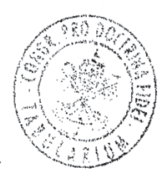




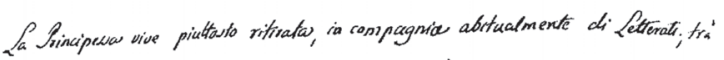

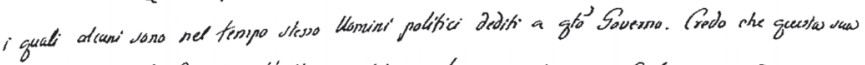

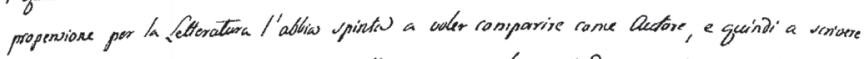

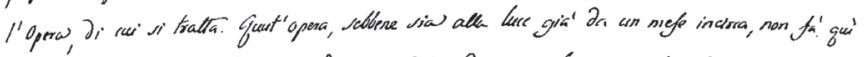

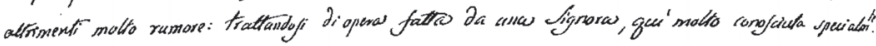

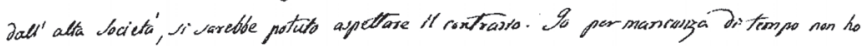

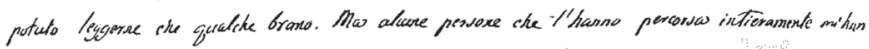

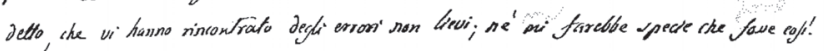

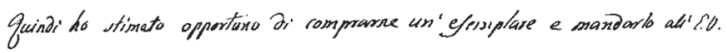

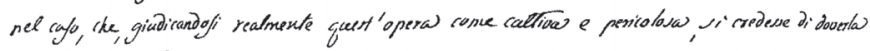
condassare pubticanente.

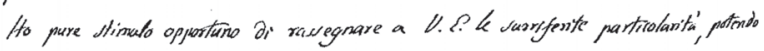
por avventuras \& med? servirye di grable normo.

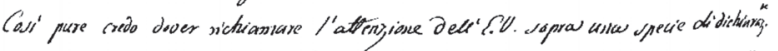

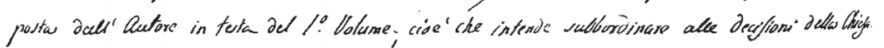
Le opincioni emesse net suso libro.

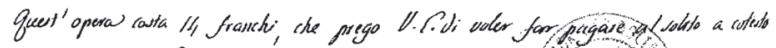
mio Agente Signor Cour. Pien- Riru: 

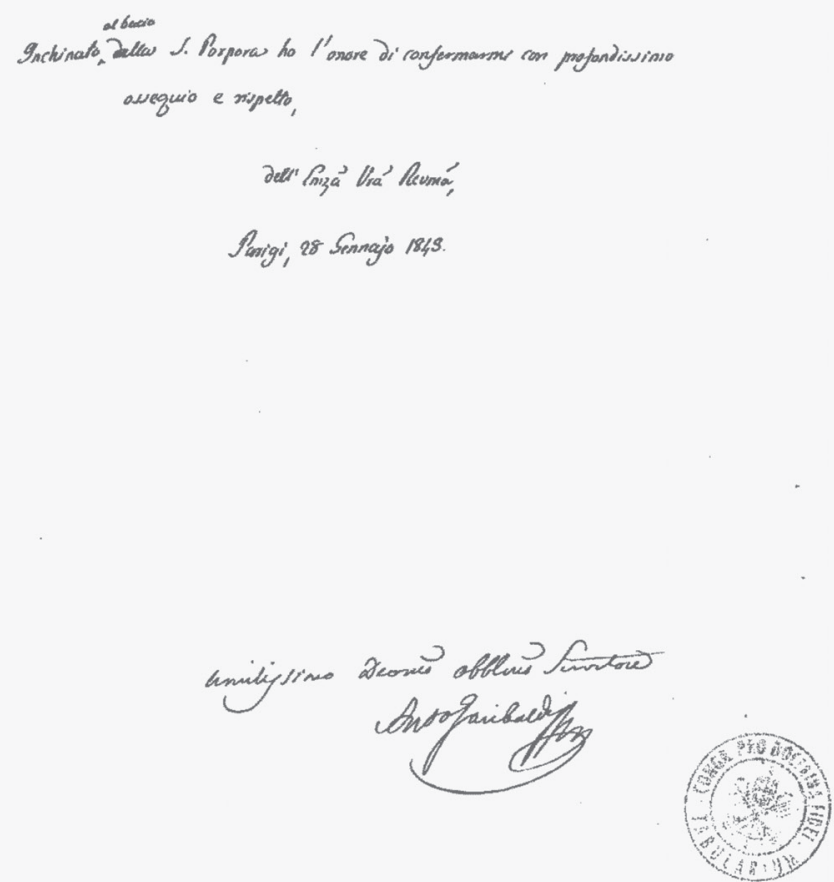


\section{Eñi Principi}

L'Opera sulla quale ho l'onore di inviare alle EE. LL. RR alcune brevi osservazioni, venne dinunziata alla ch. m. del Card. Giustiniani già Prefetto di questa S. Congr. ed accompagnata con una lettera del seguente tenore: "Ho l'onore di trasmettere qui annessa all'Emza. V. Rma una opera in due volumi che, da quanto sento, può meritare censura: il solo titolo della medesima è almeno singolare: Essai sur la formation du dogme Catholique. L'opera non ha nome di Autore, ma si sa qui notoriamente di chi è; né chi l'ha scritta e pubblicata lo dissimula. L'autore adunque di siffatta opera è una Signora ancora giovane, una Signora Italiana, appartenente alla primaria Nobiltà di Milano, cioè la Signora Principessa di Belgiojoso, nata Trivulzi. È persona di molto spirito ed istruzione: non può dirsi che sia irreligiosa sebbene in religione, come pure in politica, abbia sovente date prove di capo alquanto stravolto... ella vive piuttosto ritirata, in compagnia abitualmente di Letterati; tra i quali alcuni sono nel tempo stesso uomini politici dediti a questo governo. Credo che questa sua propensione per la letteratura l'abbia spinta a voler comparire come autore, e quindi a scrivere l'opera, di cui si tratta. Quest'opera, sebbene sia alla luce già da un mese incirca, non fa qui altrimenti molto rumore: trattandosi di opera fatta da una Signora qui molto conosciuta specialmente nell'alta società, si sarebbe potuto aspettare il contrario... credo dovere richiamare l'attenzione dell' E. V. sopra una specie di dichiarazione posta dall'autore in testa del I vol.; cioè che intende subordinare alle decisioni della Chiesa le opinioni emesse nel suo libro... Parigi 28 genn. 1843.

La dichiarazione di cui la lettera fa menzione, è la seguente: "Pénétré du plus profond respect non seulement pour la morale Chrétienne, mais pour l'institution divine et pour la conduite inspirée de l'église Catholique, j'aurais mal rendu ma pensée si chaque mot de ce livre n'exprimait la soumission la plus entière à ses décrets. J'ai cru avant même de connaître". Pag. 11. Queste parole esprimono certamente i più lodevoli sentimenti; ma ciò che segue immediatamente dopo sembra alquanto confuso: "Mais en lisant l'histoire de l'établissement de notre religion, en apercevant les traces du rare génie des premiers Pères, et celle de la prudence des églises, en voyant ceux-là choisir parmi les croyances vieilles les parcelles de vérité que Dieu a accordées de tout temps à l'humanité, les épurer, les rassembler et leur donner le complément nécessaire; et celles-ci appelées à juger du mérite de ces travaux, agir avec une sagesse constante, et ne se prononcer jamais ni vainement, ni avec précipitation, j'ai quitté la foi aveugle pour recevoir, de la connaissance, une foi non moins entière". Che cosa intende qui l'autore col dire: Vedendo questi (i Padri) scegliere tra le credenze invecchiate, le particelle di verità che Iddio ba accordato in ogni tempo all'umanità, purgarle, radunarle e dar loro il compimento necessario? Forse ch'Egli allude qui al titolo della sua opera: Essai sur la formation du dogme catholique, cioè, che la formazione del domma cattolico sia il risultato di quelle particelle di verità, scelte tra le credenze invecchiate e da' padri radunate e ridotte a necessario compimento? E poi, è egli ben vero che le Chiese chiamate per giudicare del merito di quei lavori, agirono sempre con saviezza costante, e che non pronunciarono giammai in vano, e con precipitazione? Ciò si può dire, anzi si deve dire della Chiesa universale, e del suo Supremo Capo; ma applicarlo alle Chiese particolari, questo è un esprimersi per lo meno con poca esattezza.

L'autore manifesta anche i suoi sentimenti colle seguenti parole "Quelle que soit ma répugnance à parler encore de moi, je dois dire, quoique cela me semble superflu, que mes propres opinions, renfermées dans les limites que l'Église a posées, ne se maintiendraient pas un seul instant devant les arrêts que l'Église pourrait rendre encore. Si quelque chose, dans ce livre, a pu paraître signifier le contraire, je le regrette dés à présent". Lasciando adunque a parte l'intenzione dell'Autore, e considerando soltanto l'opera in sé stessa, io la trovo assai difettosa, e scritta come da persona, a cui 
mancano le più necessarie cognizioni per trattare accuratamente di materie teologiche; anzi sembrami che si deve attribuire alla mancanza stessa di tali cognizioni quella specie di presunzione colla quale l'autore parla così francamente delle questioni le più difficili, come si vedrà da alcuni passi dell'opera, che riferirò in appoggio della mia censura.

[E qui il censore riporta ampi brani dell'Introduzione, commentandoli adeguatamente, N.d.R.]

\section{INTRODUZIONE}

“Nous aurons souvent à répéter que le Christianisme n'est pas sorti tout a coup de la Judée, tel que nous le voyons aujourd'hui, (ecco insinuata già qui la formazione del domma cattolico), et il suffira peut-être, pour donner à cette proposition un sens orthodoxe, d'ajouter qu'il était contenu tout entier dans le pages sacrées, des évangiles, (ma ciò non basta, giacché sembrasi qui escluso il verbo divino non scritto, ossia la divina tradizione) et que tant de siècles n'ont été employés qu'à l'en tirer au moyen des interprétations données ou reçues par l'église, juge infaillible en ces matières". pag. 1.

«Le dernier des évangélistes... dont la pensée, dès longtemps exercée aux abstractions orientales, entrait plus aisément dans les mystérieuses profondeurs du dogme nouveau, n'écrivit etc.». Chi sa, se per scrivere il suo Vangelo, non fosse una condizione necessaria per S. Giovanni l'essersi da lungo tempo esercitato nelle astrazioni orientali! pag. 2.

"Le maître (Jesus-Christ) avait entrepris de changer les cœurs, en s'engageant à démontrer que cette transformation nécessaire était en même temps suffisante au salut de la famille humaine; l'apôtre entreprit le premier de renverser les coutumes établies et de changer les mœurs" pag. 4. Da questo passo sembrerebbe che S. Paolo abbia perfezionata la dottrina di G.C., il quale, secondo l'autore, si contentava della sola trasformazione del cuore, e che S. Paolo vi abbia aggiunto il cambiamento dei costumi. Si vede, che qui si vuol applicare anche alle cose di religione il moderno sistema del progresso. Ma, è egli ben vero che Gesù Cristo si contentò della sola trasformazione del cuore; o per dir meglio, la trasformazione del cuore non importa essa necessariamente seco ancora il cambiamento dei costumi?

Se si dovesse scrivere una critica esatta di tutto ciò che merita censura, ne riuscirebbe un'opera molto più voluminosa di quell'autore: io mi contenterò di citare qua e là alcuni passi, i quali, a parer mio, basteranno per formare un giudizio di tutto il resto, e perciò sufficiente allo scopo di cui si tratta.

Alla pag. 39: “à l'avènement de Sévère en 192. Le repos durait déjà depuis longtemps pour eux (les Chrétiens) et ils en avaient profité pour s'étendre et pour s'éclairer. L'esprit Payen s'affaiblissait de plus en plus. L'esprit chrétien au contraire avait toute l'énergie de la jeunesse, l'assurance de la conviction, la chaleur de l'enthousiasme. Des écoles de lettres chrétiennes avaient remplacé les écoles de philosophie; les pères de l'Église, les écrivains profanes, la chaire, la tribune”. Ancorché tutto ciò che qui asseriscesi dall'autore fosse vero, si potrebbe ben addursi per provare il progresso di una istituzione umana, ma non mai per spiegare il progresso e la propagazione della religione di G.C.

Nella pag. 45: "Il (Clément d'Alexandrie) traite avec mépris les dieux de bois et de pierre, et leur oppose la simplicité du culte chrétien, qui n'admet et qui n'bonore aucune image". Non si sa capire per qual motivo l'autore abbia scritto con lettere corsive queste parole: il quale (il culto cristiano) non ammette né onora alcuna immagine.

Alla pag. 46, dopo aver riportata una lunga descrizione dei corrotti costumi dei cristiani di Alessandria del tempio di Clemente, l'autore fa il seguente riflesso: pag. 52 "Voilà donc quelle était la vie de ces chrétiens du II siècle dont nous nous faisons 
une idée si pure!". Applicando così a tutti li fedeli del II secolo, ciò che Clemente dice d'una porzione di fedeli di Alessandria.

Alla pag. 101 l'autore cita dal III lib. De' Principj di Origene, Cap. VI, questo brano: "L'Écriture dit que le dernier ennemi, qui est la mort, sera détruit... Il faut entendre par ces mots, non pas que la substance de l'ennemi, substance créée par Dieu, puisse jamais périr, mais que la volonté opposée à Dieu, laquelle procède de la créature et non de Dieu même, cessera d'exister. L'ennemi sera donc détruit, non pas de telle sorte qu'il cesse d'être; mais qu'il cesse seulement d'être ennemi" ... veggiamo ciò che vi aggiunge l'autore. Egli dice così: "Ce passage nous explique la protestation adressée par Origène à ses amis d'Alexandrie. La substance du démon étant créée par Dieu ne pourrait être détruite, mais sa méchanceté, procédant de sa propre volonté, devait cesser d'être, et la qualité de démon ou d'ennemi de Dieu, après avoir été consumée dans le feu éternel, c'est-à-dire par l'ardeur de l'amour divin". Non so se Origene abbia veramente scritto che il fuoco eterno sia l'ardore del divin amore; ma se si riflette a ciò che dice l'autore alla pag. III sembra ch'egli stesso ammetta questa dottrina; ecco le sue parole: "Il est vrai que quelques-unes des opinions que j'étais habitué à considérer comme catholiques révoltaient ma raison et me semblaient mal s'accorder avec l'infinie miséricorde de Dieu. Mais, en étudiant de près la formation du catholicisme, et en examinant la marche de l'Église, je découvris avec satisfaction, je dirais presque avec transport, que, malgré le préjugé universel, l'Eglise n'avait point porté de jugement définitif sur ces matières".

Alla pag. 140 "L'impérieux et irascible Tertullien ne convenait pas à une époque où le christianisme était proscrit et où la société chrétienne n'avait point encore adopté la forme monarchique". Proposizione del tutto conforme al sistema del progresso, o a quello della formazione del dogma cattolico. Eccone un'altra affatto simile alla pag. 142: "Plus tard, lorsque toute l'autorité de l'Église fut tombée dans les mains du Pontife de Rome".

Ma il più bel saggio dell'autore sulla formazione del domma cattolico si trova alla pag. 148 e segg.: "Bien des siècles avant la venue de I.C., l'Asie mineure, la Perse, l'Egypte et la Palestine avaient vu naître divers systèmes de cosmogonie. Le zendavesta des Persans, les écoles platoniciennes des Grecs, les mystères des Égyptiens et la Kabbale des Juifs, tous les systèmes avaient entre eux plusieurs points d'une frappante analogie". Dopo aver esposto tutti questi sistemi l'autore prosegue: "Au milieu de ces doctrines philosophiques, une religion nouvelle s'établit tout à coup. Celle-ci admit ce qu'il y avait de plus positif dans les anciennes croyances, rejeta tout ce qui était obscur et superflu, remplaça le raisonnement par la foi, expliqua les mystères par ses miracles, personnifia les abstractions, obéit enfin au caractère du peuple où elle avait pris naissance. Je veux parler de la religion chrétienne". Non v'ha dubbio che questo non sia il principio della formazione del domma Cattolico, che andò sviluppandosi poco a poco, giacché, siccome osserva l'autore alla pag. 158. "Les apôtres, occupés de fonder des églises, de prêcher l'évangile, de former les mœurs et de se soustraire ou de résister aux persécutions, les apôtres n'avaient eu ni le temps, ni l'occasion d'établir d'une manière bien précise les dogmes de la cosmogonie, de l'anthropologie, et de la théogonie chrétiennes. Mais la nécessité de combattre fit trouver plus tard des armes aux chrétiens ... en combattant les gnostiques des gentils, les chrétiens se rapprochèrent des lois juives et des codes sacrés que $S$. Paul avait paru assez disposé à abandonner, car ces gnostiques rejetaient les prophètes. Et ceux-ci avaient annoncé la venue de I.C. en combattant les gnostiques de tous les pays, ces redoutables adversaires du christianisme des premiers siècles, les orthodoxes parvinrent peu à peu à compléter l'ensemble de leurs doctrines, et à former une puissante et rigoureuse déduction des vérités si simples annoncés par Jésus-Christ. Ces conséquences logiques mais inexplicables furent acceptées par tous les orthodoxes qui composaient 
l'Église: ils les imposèrent sans les comprendre ni les développer; ils attachèrent le salut des âmes à une aveugle et volontaire soumission de l'esprit; ils condamnèrent les doutes, interdirent les recherches, et dès lors le christianisme réalisé s'annonce comme le fondateur et l'organisateur de la société nouvelle". pag. 159. Sarebbe cosa affatto superflua il voler aggiungere alcune spiegazioni ad una esposizione così chiara della maniera con cui si formò il domma cattolico.

Alla pag. 238, parlando di S. Benedetto, e dei suoi monaci, l'autore, dice che "Bien loin d'accorder à la pensée l'indépendance du corps dont elle jouissait en Orient. S. Benoit ne lui permet plus de s'exercer de peur qu'elle ne s'égare. Le moine est tenu d'obéir sans s'inquiéter si l'ordre qu'il exécute est ou n'est pas selon les lois de la justice de Dieu, persuadé qu'il doit être que Dieu a ratifié l'abdication de son jugement, et qu'il ne demandera compte qu'à son supérieur des actions que celui-ci lui a fait commettre".

Alla pag. 295 l'autore chiama S. Atanasio il fondatore del domma della SS. a Trinità, "car en le formulant il a donné un corps à l'esprit, et une forme à la pensée fondamentale du christianisme".

Alla pag. 380 l'autore termina il primo tomo della sua opera colle seguenti parole: "En racontant les troubles dont Arius fut la cause, je n'ai pus cru devoir suivre l'exemple des historiens de l'Eglise, qui tous s'accordent à présenter cet hérésiarque sous les traits les plus odieux. Je n'ai vu dans Arius qu'un esprit novateur mais soumis, égaré mais repentant. Je ne saurais d'ailleurs me défendre d'un sentiment d'intérêt pour ces hommes qui vivent par l'intelligence, qui emploient toute leur activité et leur force au service d'une idée qu'ils croient fondée, et qui placent leur espérance et leur bonheur dans son triomphe et son établissement dans le monde. L'on sert mieux d'ailleurs l'esprit de vérité en disant ce qui semble vrai qu'en accablant d'invectives et de calomnies ceux que leur aveuglement dérobe à son éclat". Questo sol passo giustifica ciò che si dice dall'autore nella lettera di denunzia, cioè ch'egli abbia sovente dato prove di capo alquanto stravolto. Ne troveremo altri esempi nel

\section{TOMO II.}

Alla pag. 21 e seg. l'autore descrive il carattere di Giuliano l'apostata, e tra le molte altre buone qualità, dice di lui: "Julien était doux, j'allais dire charitable... il était sobre et aimait la pauvrété... Malgré sa jeunesse, les conseils de ses amis et l'intérêt de l'empire, il ne voulut plus se remarier, et vécut toujours de la manière la plus chaste. Sa fortune ne l'éblouit point, et il était si peu attaché à la vie que lorsqu'à la fleur de l'âge il fallut la quitter, il en remercia les Dieux et se déclara satisfait. ... Sa mort fut plus belle qu'il ne devait l'attendre; car il vécut en homme habile, et morut en sage. À ses derniers instants il se rappela et la droiture de ses intentions et la douceur de ses mesures, sa chasteté, son amour des dieux, sa sobriété, ses goûts studieux, son respect pour les philosophes et il se rassura. Se souvint il aussi des taches qui obscurcirent sa vie? Si sa mémoire lui rendit ce triste service, elle ne parvint point à troubler la sérénité de son âme. Chacun connait les admirables paroles qu'il prononça en mourant, et auxquelles on ne peut reprocher qu'un peu de langueur et d'apprêt. Il s'humilia devant la pensée de ce qu'il avait pu faire et il se confia dans la bonté de Dieu universel. Jeune et puissant, il rendit la vie sans murmurer au maître qui la lui redemandait. Il en adora les décrets qui le frappaient et qu'il ne comprenait pas. Le Dieu qui inspirait de tels sentiments pouvait il ne pas être le Dieu des chrétiens?"

Alla pag. 82 "Saint Ambroise n'était encore âgé que de 57 ans; mais sa carrière était terminée. Il avait élevé l'épiscopat jusqu'auprès et quelques fois au-dessus du pouvoir impérial. Il avait contribué à établir la supériorité de l'église d'occident, sur celle d'orient, et la préminence des habitudes positives de l'une sur l'esprit spéculatif de l'autre". 
Alla pag. 87, capo III, in cui si tratta principalmente di S. Girolamo, l'autore comincia così: “A mesure qu'en s'éloignant de son berceau, le christianisme acquiert la puissance et les richesses du monde, on le voit perdre en partie son caractère divin et tomber dans l'humanité. C'est un bien triste spectacle que celui de ces hommes appelés encore saints seulement parce qu'ils succèdent à des saints, qui ne renoncent qu'au bonheur, et qui se livrent sans scrupule aux passions haineuses parce qu'elles ne leur apportent aucune joie. Ils se quittent lors qu'ils s'aiment, et se cherchent lorsque la haine a remplacé l'amitié. L'on trouve encore et toujours dans l'histoire du christianisme des persécuteurs et des persécutés; mais les premiers se disent chrétiens et les seconds ne pardonnent pas. Ils se vengent, tantôt par des violences brutales, tantôt par d'adroites calomnies... de savants rhéteurs s'acharnent sur les écrits nouveaux, espérant y découvrir quelque parole obscure qu'ils convertiront en hérésie, et contre laquelle ils feront éclater leur éloquente colère. Les dogmes chrétiens deviennent chaque jour plus précis, mais le sentiment chrétien dont J. C. a fait la principale condition du salut des hommes se perd et s'oublie”... Egli risulta dal contesto di tutto questo capo che tutti quei riflessi si debbano principalmente applicare a S. Girolamo ed agli altri padri di quei tempi.

Alla pag. 103: "Pour combattre Jovinien, Saint Jerôme se jeta dans l'excès contraire, et, non content de condamner les secondes noces, il traita les premières avec tant de mépris, en les comparant à l'état de virginité, que Rome entière se souleva contre lui et se félicita de l'avoir expulsé de ses murs".

Alla pag. 110: .... "Mais pour réussir dans cette entreprise, il eût fallu faire convenir S. Jérôme de ses torts, et cela était au-dessus de toute puissance bumaine..." Pag. 112: "Il semblait que les malheurs eussent seuls le privilège d'imposer un court silence aux sentiments haineux qui remplissaient le cœur de S. Jérôme. Mais l'on eût dit aussi qu'en cessant de combattre, S. Jérôme cessait de vivre. Accablé par les austérités et par les maladies, son corps était soutenu, non par la pensée, mais par les passions, et tombait sans force dès que celles-ci perdaient leur aiguillon ou leur liberté..." Pag. 131: "Ces disputes et ces traductions n'occupaient pas tellement S. Jérôme, qu' il n' eût encore le temps d'entretenir d'autres discordes..." Basteranno certamente questi pochi passi per far conoscere con che rispetto il nostro Autore parla dei SS. Padri e Dottori della Chiesa Cattolica. Egli parla anche molto nella sua opera degli Ariani, Manichei, Donatisti, Pelagiani ed altri eretici dei primi secoli, dei loro errori, e dei Santi Patri che li impugnarono; ma siccome io mi sono già troppo esteso, mi contenterò di riferire ancora alcune brevi citazioni che non hanno bisogno di alcun Commentario.

Alla pag. 178 parlando dei Donatisti, dice: "Ils disputaient peu; et sur quoi auraient-ils disputé, puisque leurs dogmes ne différaient en rien de ceux des Catholiques? Mais ils calomniaient, ils injuriaient, ils tuaient". Alla pag. 190 "S. Augustin s'y montre (nei libri del libero arbitrio) peut-être plus que partout ailleurs, éclairé parfois par de sublimes lumières, et égaré souvent par la minutieuse subtilité de son esprit. Il veut établir l'existence du libre arbitre, mais il ne croirait pas en être venu dignement à bout s'il n'y avait employé l'argumentation. Il ne sent pas combien sont belles les vérités qui ne demandent ni ne supportent la démonstration. Qu'est-ce qui est plus au pouvoir de la volonté que la volonté même? s'écrie-t-il. Personne n'est assez extravagant pour oser dire que nous ne voulons pas volontairement. Par de pareils jeux de mots..." Ed alla pag. 192: "Nous verrons plus tard. S. Augustin revenir à ces argumentations, rentrer dans ce cercle vicieux ...". Pag. 193 "Lorsqu'il nous parle du péché et de sa puissance, il semble avoir oublié ce qu'il nous a dit dans ses Soliloques...".

Pag. 195: "La question de la prescience divine occupe encore S. Augustin, et il ne semble pas heureux dans ses efforts pour la concilier avec la liberté bumaine". Pag. 201: "S. Augustin écrivit deux ouvrages dont l'un porte pour titre: Du bien du ma- 
riage, et l'autre: de la Sainte Virginité. Il expose également dans les deux que l'union des sexes a été le premier péché d'Adam; que les désirs de la chair en sont la conséquence et la punition c'est-à-dire le péché originel avec lequel nous naissons tous; que le mariage en est le remède permis; que la virginité est pour l'âme l'état de bonne santé".

Alla pag. 268: " S. Augustin trouvait extravagant de prétendre que Dieu punissait dans les enfants les péchés qu'ils auraient commis s'ils avaient vécu. Il pouvait avoir raison. Mais il trouvait que Dieu punissait en eux un péché qu'ils n'avaient jamais commis et qu'ils ne commettraient peut-être jamais. Cela valait-il beaucoup mieux?"

Alla pag. 274: "Il (Pelage) attribuait aux Catholiques des opinions que jamais aucun d'eux n'avait professées, comme par exemple: 'Que le libre arbitre avait péri', proposition que l'Église n'a jamais approuvé tout en adoptant les principes dont elle était la conséquence"

Alla pag. 280: "S. August. conclut que tous les hommes sont morts en Adam, de ce que I. C. est mort pour les racheter tous, nous les verrons plus tard se contenter de garder cette conclusion, tout en repoussant le principe d'où elle émane".

Benché nel trascrivere tanti passi, io mi avvedo di essermi esteso più del necessario per far conoscere il merito di quest'opera, ciò nondimeno essi non sono che una minima parte di quelli che aveva notati nel leggerla, e che mi sembravano degni di censura. Che se si voglia ammettere la buona intenzione nel descriverla, pure non si può far a meno di accusarlo di presunzione per aver voluto trattare materie delle quali non aveva le necessarie cognizioni. Quanto all'opera stessa, sebbene a parer mio meriti di essere riprovata, ciò nondimeno, poiché non sembra fare alcun rumore, non le darei quella specie di importanza che risulta da una sola solenne e pubblica condanna; bensì per togliere dalla mente dell'autore la voglia di comporre altre opere, l'avviserei che il motivo per cui non viene vietata ai fedeli la lettura di questo suo primo lavoro è appunto quello, perché di fatto essi non lo leggono.

Sottometto però il mio giudizio alla saviezza delle EE. LL. R me e baciando loro la S. Porpora con profonda venerazione ho l'onore di raffermarmi.

Uño e Dm̃o Servitore Agostino De La-Croix della C. Di Gesù Cons. ${ }^{24}$

(24) Abbiamo ritenuto opportuno trascrivere una copia dell'originale di non facile lettura, rico- struendo anche le parole mal trascritte o incomprensibili. 


\section{I giudizi su "L'Essai sur la formation du dogme catholique"}

È molto difficile rendersi conto di quale sia stata l'impressione suscitata nel pubblico dall'Essai, pur essendo necessario distinguere quella manifestata dai suoi pochi e scelti lettori da quella, suscitata nei molti, che conobbero soltanto il titolo. Ė incontestabile che l'opera fu giudicata degna di essere discussa ma, più che altro suscitò grande sorpresa e non poca ammirazione per sé stessa, cioè come uno straordinario sfoggio di erudizione da parte di un'aristocratica del bel mondo.

Fra gli italiani di Parigi la pubblicazione della nobildonna letterata destò un grande stupore: i più malevoli non sapevano quale giudizio esprimere. Alcuni si rallegravano che un'italiana facesse favorevolmente parlare di sé a Parigi, pur rammaricandosi che quell'italiana fosse proprio la Belgiojoso. Ma ci fu chi comprese il valore dell'opera, Costanza Arconati, donna colta e frequentatrice dei salotti intellettuali, che, in una lettera ad Antonio Trotti del 5 ottobre 1842, scrive: «Qui (a Parigi) il pubblico l'ha molto favorevolmente accolta ed è certo che è scritta con raro talento» ${ }^{25}$.

I pareri espressi nell'ambiente aristocratico sono spesso divertenti, soprattutto per la loro forma retorica che tradisce la presuntuosa ignoranza dei loro autori. La duchessa di Talleyrand, scrivendo al Barante, dice che il titolo dell'opera della Belgiojoso le sembra eretico perché esprime l'idea che il dogma cattolico possa essere "formato". La duchessa che ignorava la storia della chiesa, la laboriosa opera dei Concili, delle lotte del papato contro le eresie, si immagina che i dogmi del cattolicesimo siano usciti bell'e fatti, non si sa poi da dove: «La princesse Belgiojoso a, dit-on, des habitudes très religieuses et comme elle est excessive en toutes choses, on dit qu'elle a déjà adopté un costume presque monacal. Avez-vous lu un livre de théologie qu'elle vient de publier et qui a pour titre Des Dogmes du Catholicisme (sic) dans les premiers siècles de l'Église? Ce titre ne parait pas très orthodoxe car le dogme est de tous les siècles et ne saurait varier comme la discipline. On dit cependant le corps de l'ouvrage plus correct que le titre» ${ }^{26}$.

Un'altra signora, la Vicomtesse de Janzé, accennando alla Belgiojoso in un suo studio su Alfred de Musset e sapendo che era stata in Terrasanta nel 1852, afferma che vi era andata per raccogliere gli elementi per scrivere il suo libro sul Dogma (pubblicato dieci anni prima) quasi che le notizie intorno allo sviluppo della teologia cattolica si potessero raccogliere in Palestina e non altrove ${ }^{27}$.

Questi giudizi e questi pettegolezzi di dame aristocratiche dimostrano quanto Cristina fosse loro superiore per cultura ed intelligenza e giustificano la sorpresa e l'ammirazione che destò l'opera in coloro che erano in grado di fare confronti. Un giudizio più accettabile è quello espresso da Hortense Allart nel 1842. Prima ancora di avere terminato di leggere il libro scriveva al Sainte-Beuve: «Mais ce n'est pas un livre, elle (l'autrice) n'a pas d'opinion ni de but ${ }^{28}$.

In realtà l'opera di Cristina non era altro, almeno nelle sue intenzioni, che un saggio di divulgazione all'apparenza estremamente circospetto.

La stessa autrice aveva dichiarato iniziando la sua opera: «Ce livre ne sera ni une histoire ni un traité, car il manque d'ordre et de suite dans l'arrangement des faits, aussi bien que de profondeur dans l'exposition et dans l'examen des doctrines. [...] Écrites à mesure que l'étude m'en fournissait les matériaux, ces pages ne devaient

(25) A. MalvezzI, Il Risorgimento italiano in un carteggio di patrioti lombardi, Milano, Hoepli, 1921, p. 45 .

(26) Barante, Souvenirs, Lett. Rochecotte, 4 novembre 1842 , p. 42.

(27) La Vicomtesse de JanZé, Études et récits sur
Alfred de Musset, Paris, Plon Nourrit, 1891, p. 188.

(28) H. Allart, Lettres inédites à Sainte-Beuve (1841-1848) avec une introduction des notes, éd. Léon SÉché, Paris, Société du Mercure de France, 1908. 
servir qu'à aider ma mémoire, et n'être lues que par un petit nombre d'amis indulgents $\gg^{29}$.

Il Sainte-Beuve espresse un'opinione lusinghiera tanto più importante in quanto non solo riguarda lo stile letterario dell'Essai, ma anche perché l'autore di Port Royal aveva una particolare conoscenza delle questioni religiose: «Il a paru un livre - scrive - encore inachevé (2 volumes de la princesse Belgiojoso sur la Formation du Dogme Catholique) c'est sérieux, catholique d'intention, semi-pélagien et origénien de fond, d'un style très ferme, très simple, enfin une très précieuse curiosité venant d'une italienne galante, d'une Trivulce. Son nom n'y est pas, mais elle l'avoue» ${ }^{30}$.

Riconoscimenti sullo stile giungono anche dal filosofo Felix Ravaisson che apprezza soprattutto «la présence vivifiante d'une âme, et l'âme d'une flamme»» ${ }^{31}$, e Alexis de Tocqueville: ammira anche lui il suo modo di esprimersi: «Vous avez retrouvé les styles du beau temps de notre littérature, ce qui est assurément merveilleux» ed apprezza quanto sia vero che il cristianesimo, ai suoi inizi, abbia subito l'influenza del genio e delle abitudini intellettuali e morali dei diversi paesi che conquistava ${ }^{32}$.

Nicolò Tommaseo le rimproverò invece di aver scritto l'opera in francese e Cristina gli risponderà che, vivendo in Francia da lungo tempo, si sentiva più padrona di quella lingua che non dell'italiano ${ }^{33}$.

La Belgiojoso fece dono della sua opera a Gioberti ed egli, sebbene l'abbia poi decisamente negato nel Proemio del rinnovamento, le scrisse cortesemente. La lettera del Gioberti è purtroppo andata perduta, ma la sua esistenza è accertata dal ringraziamento che gli indirizzò la Belgiojoso e che si trova tuttora fra le sue carte:

\section{Signore,}

condannata da una debolezza della vista ad un ozio quasi assoluto, avevo pregato l'amico Massari di ringraziarla in nome mio per la lettera che da lei ricevetti. Eppure non posso tralasciare di dirle anch'io due parole, onde tentare di esprimerle quanto mi sia riuscito caro il suffragio di persona di acuto ingegno, di molta scienza, di chiara e perciò appunto di salda ed alta fede, come io tengo (e lo tiene ognuno) ch'Ella sia.

Poche sono le odi ch'io ambisco: e fra queste stan certo fralle prime le sue.

Scusi se scrivo così laconico: ma i miei occhi non mi permettono di fare altrimenti. Gradisca, comunque sieno espressi, i sensi della mia stima e gratitudine.

Cristina di Belgiojoso ${ }^{34}$

Purtroppo è andata smarrita anche un'altra lettera alla Belgiojoso di Alessandro Manzoni, ma sappiamo dal Cantù che questo documento è esistito. «Egli (il Manzoni) - dichiara il Cantù - le scrisse una seria lettera quando ella pubblicò La Formation du Dogme Catholique». L'aggettivo "serio" è in contrapposizione a "scherzoso" e quindi si potrebbe interpretare come "severo". Secondo Manzoni non poteva spettare alla Belgiojoso di scrivere su certi argomenti $i^{35}$.

In un altro ambiente parigino, quello dei Girardin, la pubblicazione dell'opera sollevò un notevole scandalo: non bastava la concorrenza del vivace salotto della nobildonna in rue d'Anjou, né l'omaggio di certi uomini illustri e di "pericolosi" patrioti. L'intrusa italiana si metteva anche a pubblicare libri.

(29) C. DI Belgiojoso, Essai sur La Formation du dogme catholique, cit., vol. I, p. I.

(30) Correspondance inédite de Sainte-Beuve publiée par Mme. Bertrand, 28 dicembre 1842.

(31) A. MalvezzI, La principessa Cristina di Belgiojoso, Milano, Treves, 1936, II, p. 376. Senza ulteriori referenze.
(32) Ibid., p. 377.

(33) Ibid., pp. 345-346.

(34) Biblioteca Civica, Torino, Carteggio Gioberti, lett. N. 326.

(35) C. CAntu, Alessandro Manzoni, Milano, Treves, 1882, II, p. 41. 
Delphine Girardin, allarmata, sdegnata, chiamò a raccolta tutti i suoi fedeli. Accorsero il Méry, Théophile Gautier e, poiché il gruppo Girardin stava componendo un romanzetto, La croix de Berny, ciascun capitolo del quale era affidato ad un diverso scrittore, così il Gautier si assunse l'incarico di trattare l'argomento Belgiojoso: lo fece descrivendo il salotto della rue d'Anjou, che però era chiuso già da quattro anni e probabilmente lo scrittore non lo sapeva.

Poiché si trattava di porre un freno urgentemente alle velleità letterarie della Belgiojoso, svalutando la sua opera di storia ecclesiastica, mettendola in ridicolo, non si poteva far meglio che rappresentare l'autrice di quell'opera in abiti ecclesiastici e riempire il suo salotto di crocifissi, acquasantiere e quadri sacri. Questo scritto raggiunse lo scopo del gruppo. La contessa Merlin, che scriveva biglietti affettuosi alla Belgiojoso qualche anno prima, ora che aveva bisogno di un appoggio finanziario della casa editrice Girardin, contribuì alla campagna della stessa contro di lei, dedicandole qualche pagina del suo romanzo Les Lionnes de Paris, uno dei tanti precursori, assieme ai Misteri di Parigi, dei romanzi gialli odierni. In questo libro figurano parecchi italiani e, fra gli altri, il conte "Peppola" di Bologna, con relativa mortadella; la Belgiojoso appare sotto il nome di Giuditta e su di lei leggiamo questo ironico ritratto: «Elle attira chez elle les hommes les plus marquants pour l'exaltation de leurs opinions; elle causait, discutait avec eux, et les surprenait par sa rare intelligence et par les traits imprévus de son caractère singulier et viril. Encouragée, enivrée par ses succès, elle entreprit un travail sur lequel elle fonda les plus grands espérances de succès, et qui se ressentait de l'originalité de son caractère. Cette œuvre devait avoir pour titre: Moyens infaillibles d'accroître et de civiliser les populations naissantes dans les déserts qui avoisinent les Montagnes de la Lune, suivis d'un projet de constitution pour le royaume de Bambara. Le succès que cette production valut à Giuditta, la fit proclamer lionne littéraire et politique de l'époque» ${ }^{36}$.

Alfred de Musset, innamorato respinto, volle vendicarsi dedicando a Cristina dei versi che avrebbero dovuto essere un ritratto morale, intitolati Sur une morte, in cui, oltre a vedere in lei una donna fredda e inafferrabile, priva di impulsi amorosi, lasciava trasparire il suo convincimento che fosse anche incapace di pensare:

Elle pensait, si le vain bruit

d'une voix douce et cadencée

comme le ruisseau qui gémit

peut faire croire à la pensée $e^{37}$.

Questa velata e maligna allusione, scritta proprio nei giorni in cui era uscito l'Essai, dimostra l'acredine di un poeta esasperato dalla freddezza di colei che, pur negandosi, gli era sempre stata amica. I particolari della relazione Musset-Belgiojoso e di questo increscioso episodio, sono raccontati ampiamente dal Malvezzi ${ }^{38}$.

Tanti malevoli commenti contribuirono a diffondere un'interpretazione falsata della figura della nobildonna, che poi fu ripresa dagli autori delle sue biografie.

Raffaello Barbiera solleverà il sospetto che, ad aiutarla a redigere l'opera, sia stato l'amico Abbé Cœur: «Quel libro - scrive - [...] fu steso dalla principessa ma ho l'assoluta convinzione che vi ha messo mano un giovane geniale predicatore francese: l'Abbé Cœur»"

(36) Les Lionnes de Paris par feu le Prince De ..., II, p. 236.

(37) In «Revue des deux mondes», 4ème série, tome 32, 1842.

(38) A. Malvezzi, La principessa Cristina di Bel- giojoso, cit., pp. 357-370

(39) R. BARBIERA, La principessa di Belgiojoso, $i$ suoi amici e nemici, il suo tempo, Milano, Treves, 1902, p. 139. 
Mentre il Malvezzi, che ha consultato i testi manoscritti, dichiara: «Dell'opera della Belgiojoso, Essai sur la Formation du Dogme Catholique, si conservano voluminosi manoscritti delle varie stesure, tutte di suo pugno, senza neppure una postilla di calligrafia dell'abate Cœur, né d'altri corrispondenti esattamente al testo stampato» ${ }^{40}$.

Il carteggio con Mignet rivela che Cristina gli sottoponeva i suoi manoscritti perché rivedesse il suo francese, come era abituata a fare con tutte le sue opere ${ }^{41}$.

Da un esame più attento dell'Essai da parte della critica moderna, ci pare interessante, anche se limitato, il giudizio del Malvezzi che definisce l'opera della Belgiojoso «più che l'espressione di opinioni personali, un lavoro di semplice divulgazione di fatti storici, nobilissimi, esposti obiettivamente. La novità starebbe invece nell'aver interpretato la teologia, pregio del tutto femminile, con il sentimento e non con l'intelletto nel tentativo di rendere sensibile l'intellegibile» ${ }^{42}$. E qui si trova d'accordo con il Ravaisson citato precedentemente.

Diversi lettori temevano la messa all'indice dell'opera. Per il Whitehouse la parte in cui si percepisce più viva l'impronta personale dell'autrice è quella filosofica, dove si avverte piuttosto un eclettismo che una rigorosa ortodossia.

E colloca giustamente il lavoro della Belgiojoso nel moto di rinascita dello spiritualismo cristiano che alimentava la visione religiosa del progresso dell'umanità, richiamandosi ai problemi delle origini cristiane, e dell'eternità del male. Tramite la dottrina delle preesistenza dell'anima e la condanna dell'inferno, promulgata da Origene, si affermava l'ideologia del progresso indefinito, mettendo in relazione politica e religione ${ }^{43}$.

Il Santonastaso trova un'influenza di Jean Reynaud che, come Leroux, sosteneva la teoria dl progresso contrapposta a quella di autorità.

Egli afferma che l'atteggiamento della scrittrice è quello modernistico della critica alla scolastica: mediante il perfezionamento dell'infinito delle anime, mediante la liberazione del corpo, della materia, l'anima riacquista la purezza primitiva; e se, con un'acuta intuizione, conclude dicendo che la Belgiojoso si ispira agli spiritualisti riprendendo le loro teorie, non per questo la sua opera è solo di divulgazione, ma porta l'impronta delle convinzioni intime della sua autrice, espresse in uno stile raffinato ed elegante ${ }^{44}$.

E, per concludere, il giudizio più attuale e penetrante di Mino Rossi: «Nell'Essai sur la Formation du Dogme Catholique è tratteggiato un quadro di un'umanità alla ricerca della propria identità di essere uomini, cioè della propria coscienza. Il cammino verso l'affermarsi della verità è già una delle verità accessibili alla mente umana: sta nella sua ricerca, perché non esiste verità assoluta $[\ldots]$.

Il pensiero di Cristina mirando all'apice del problema che vedeva in conflitto la libera volontà e la libera coscienza con i dogmi della fede, lo risolve in conformità alla sua dottrina, primato incontrastabile della libertà di coscienza.

Nelle idee di Cristina circola anche una vena di gallicanesimo, tesa ad appoggiare una linea libertaria da contrapporre ad un magistero ecclesiastico, caratterizzato per il suo intransigente immobilismo [...]. Cristina affronta il tema delle eresie, risalendo alle dottrine dei padri, per mostrare come la duttilità dei primi secoli del cristianesimo fosse vincente, rispetto al conservatorismo attuale, bloccato nella pietra dei dogmi che non lasciavano campo al libero esame...».

(40) A. Malvezzi, La principessa Cristina di Belgiojoso, cit., p. 347.

(41) L. SEvergnini, La principessa di Belgiojoso, Edizione Virgilio, Milano, 1972, pp. 78-79.

(42) A. MalvezzI, La principessa Cristina di Bel- giojoso, cit., p. 375.

(43) H.R. WhiteHouse, Une princesse révolutionnaire, Lausanne, Payot et C., 1907, p. 91.

(44) G. Santonastaso, La società di Jean Reynaud, in «Nuova Antologia», giugno 1952. 
Mino Rossi ha soprattutto intuito che: «Fuori dal quadro europeo, fuori dalle correnti di pensiero che percorrevano l'Europa di allora a che provocavano gli studiosi ad opere approfondite ed impegnate, i lavori di Cristina potevano essere giudicati come lavori usciti dal cervello di una povera pazza... $\gg^{45}$.

Da questo momento si tornerà a parlare della donna e della patriota Cristina di Belgiojoso, in particolare nei giorni delle commemorazioni dell'Unità d'Italia, ma l'Essai sarà dimenticato.

E, dopo una decina d'anni di silenzio su quest'opera, abbiamo pensato fosse utile riportarla alla luce perché aiuta a comprendere meglio il pensiero di Cristina, perenne perseguitata, la sua cultura, la sua indipendenza spirituale, superando tanti malevoli commenti di un mondo troppo vecchio per lei. 


\section{DECRETUM}
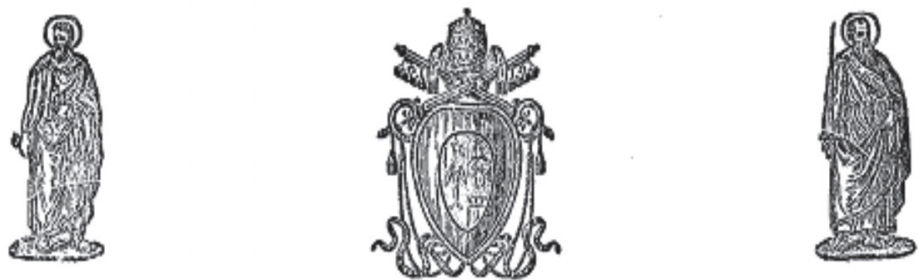

Feria V. alic 17. augusti 184s.

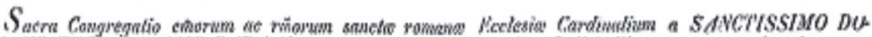

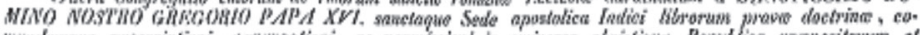

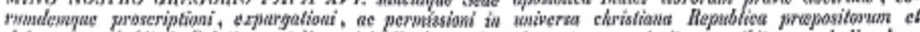

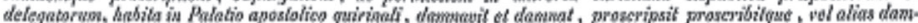

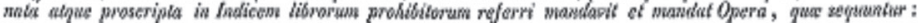

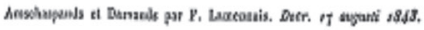

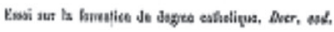

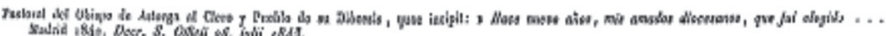

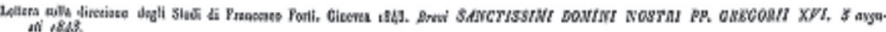

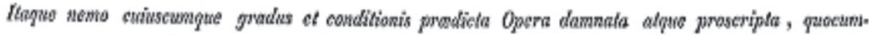
que loco, ef guocumptre idliomate, aut in posterum edere, ant edila legore, vel retinere andeat, sed beo-

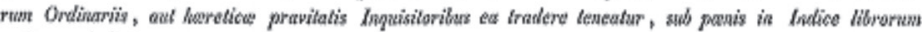
vetiluwen inclictis.

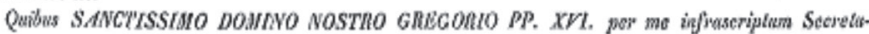
rimm rolakis, SANCTKTAS SUA Deesethm probavit, ot promalgari pracopit. In guorum futem ols.

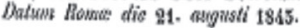

\section{A. CARD. MAIUS pRAEFECTES}

Iseo fo soess

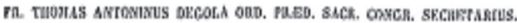

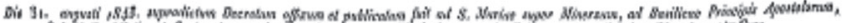

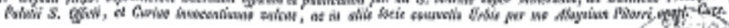

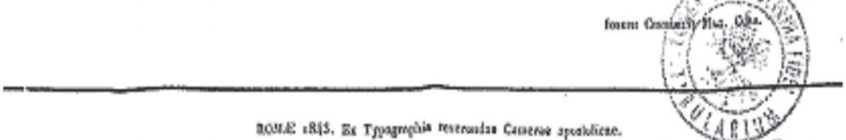

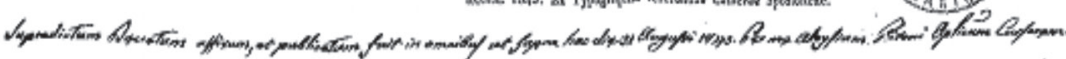



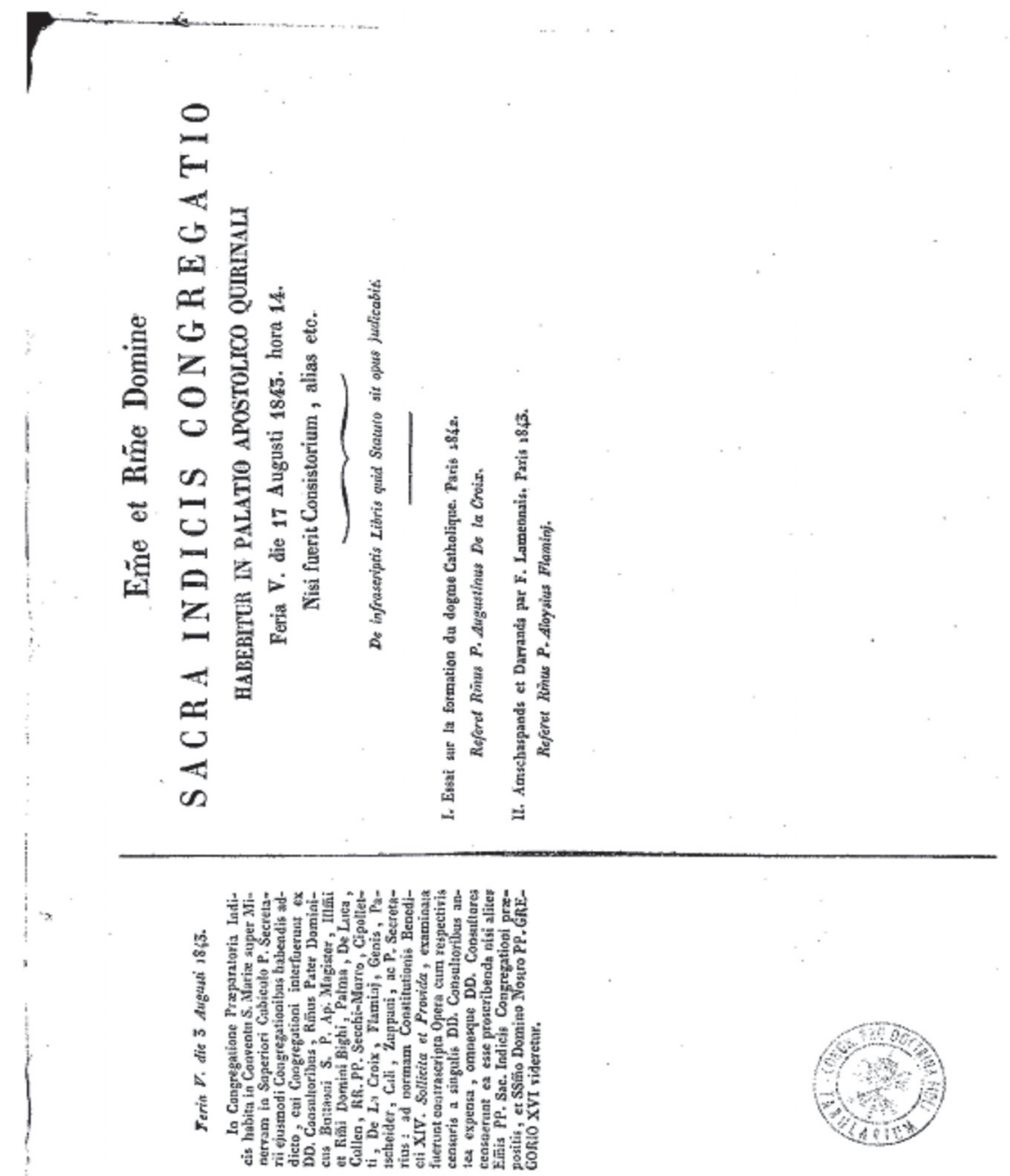
[Relazione definitiva di condanna che riprende le idee del censore De-Lacroix eliminando ogni sia pure modesta attenuante. Ė preceduta da una condanna delle opere di Lamennais]

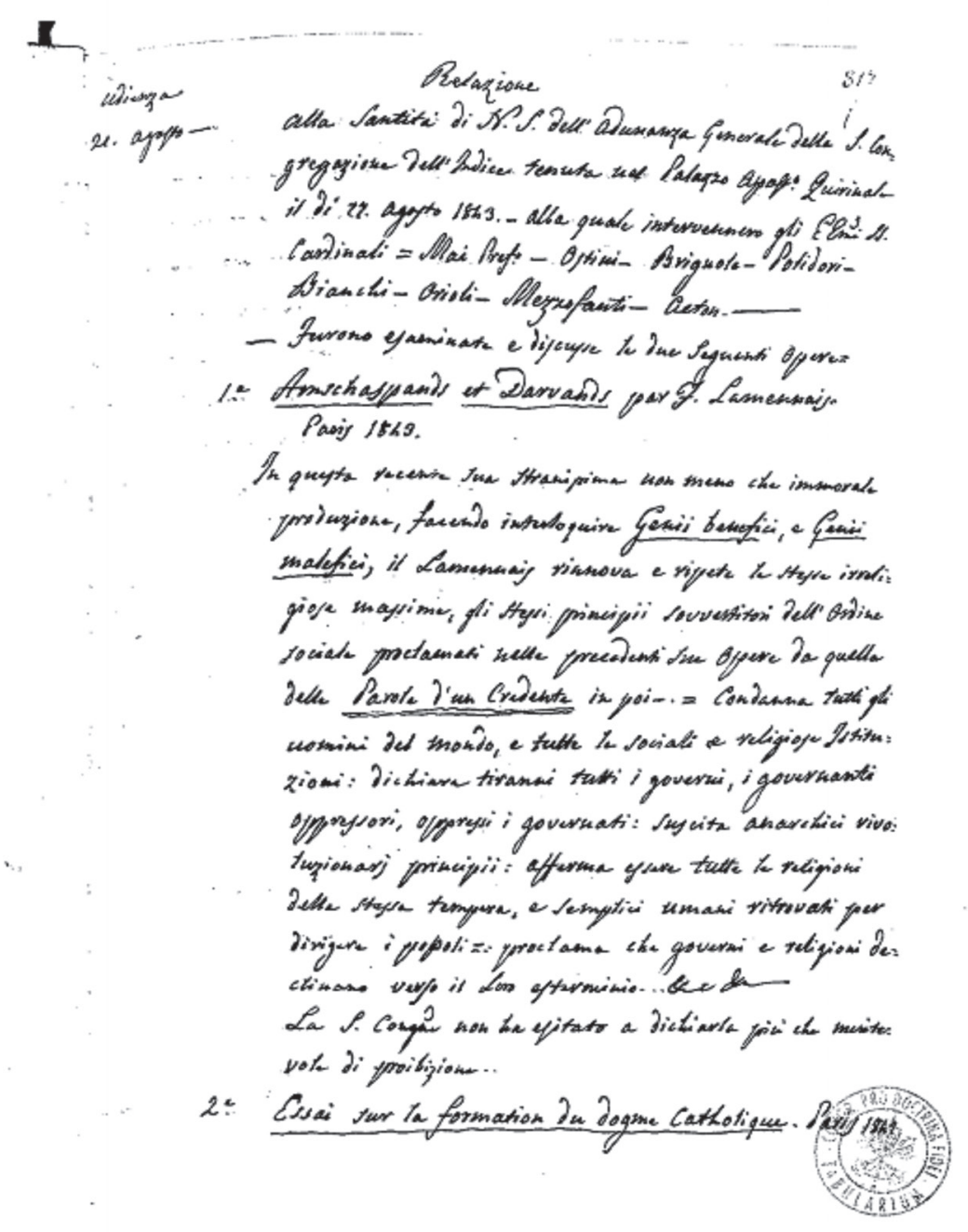




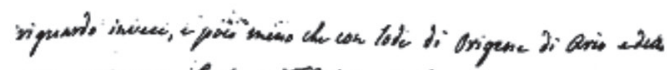

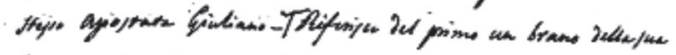

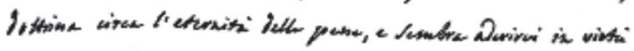

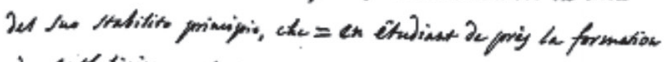

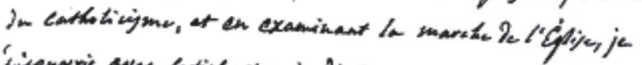

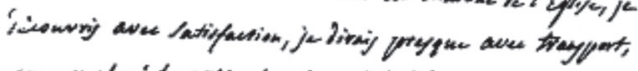

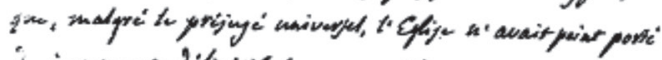

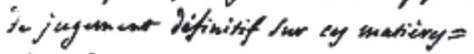

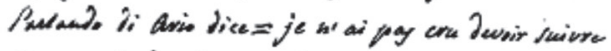

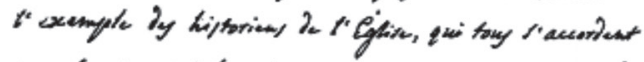

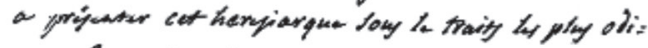

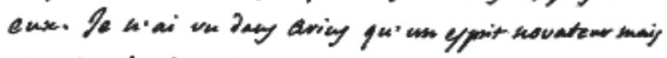

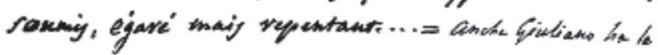

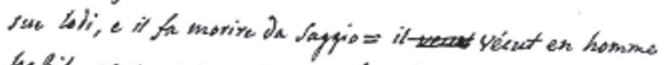
habile, at morreten sage $=$ de dender

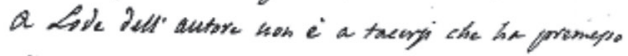

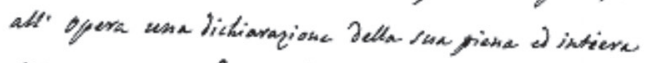

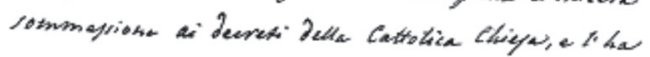
rigeriatid alle pay. $t V$.

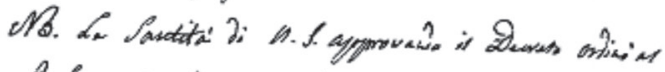

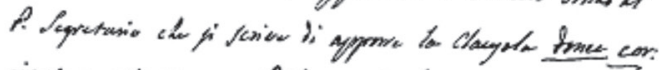

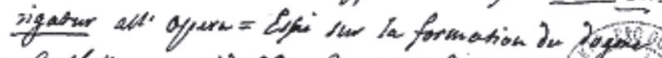

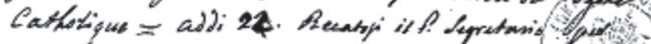




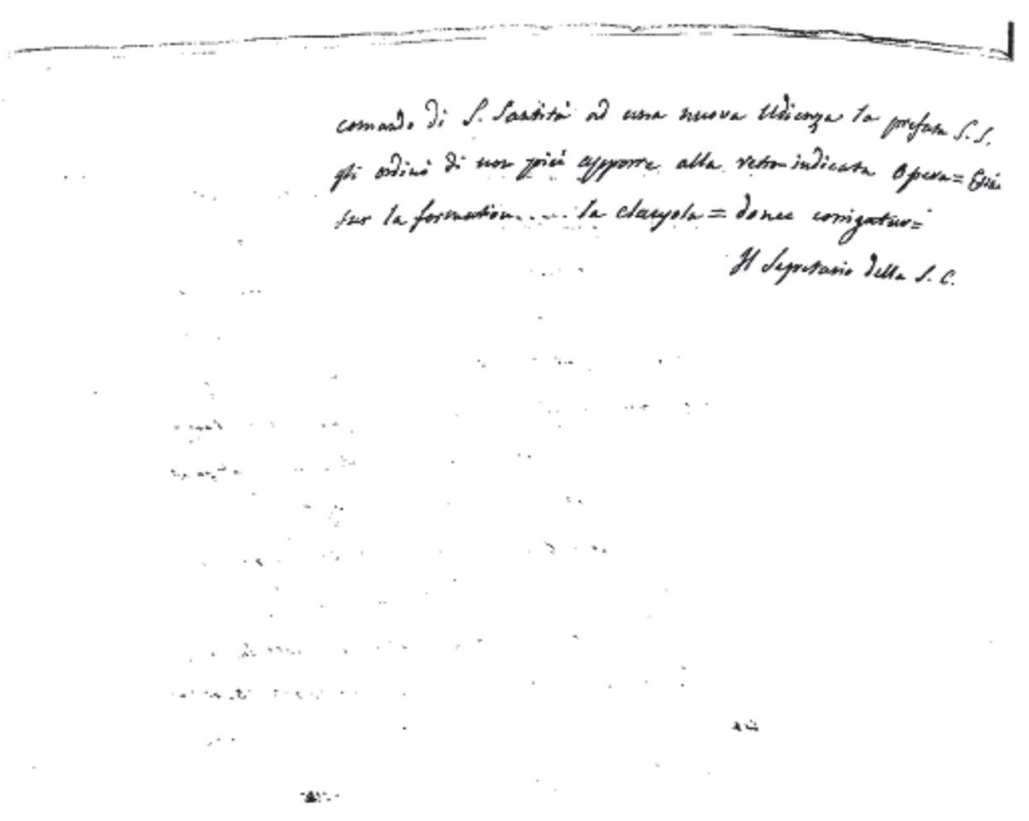

s.

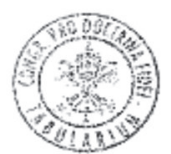

